\title{
Um Modelo para Formação e Difusão do gel formado pela Reação Álcali-sílica no Concreto
}

\author{
Fábio A. N. Balbo, Guilherme A. Pianezzer, \\ Programa de Pós-graduação em Méodos Numéricos em Engenharia \\ 81531-990, Curitiba, PR \\ E-mail: andrebalbo@gmail.com, guilherme.pianezzer@hotmail.com, \\ Liliana M. Gramani, Eloy Kavisky, \\ UFPR - Universidade Federal do Paraná \\ 81531-990, Curitiba, PR \\ E-mail: 1.gramani@gmail.com, eloy.dhs@ufpr.edu.br, \\ Marcelo R. Teixeira \\ UFPA - Campus Tucuruí \\ 868464-000 Tucuruí, PA \\ E-mail: marcelorassyteixeira@gmail.com
}

13 de Janeiro de 2014

\begin{abstract}
Resumo: Este trabalho apresenta um modelo criado para a representação da evolução da reação álcali-sílica (RAS) presente em muitas construções que estão sujeitas a umidade excessiva. O modelo é composto por duas etapas. Na primeira etapa são estudadas as reações químicas que dão origem ao gel deletério proveniente da RAS. A análise destas reações, por meio da cinética química, origina um sistema de equações diferenciais ordinárias que é resolvido afim de obter a quantidade de gel gerada a medida que o tempo passa. Na segunda etapa é criado um modelo de difusão para o gel, formado na primeira etapa, na região de transição entre agregado e argamassa. O modelo de difusão é resolvido numericamente por meio do Método de Diferenças Finitas. Como resultado obtém-se a distribuição da concentração de gel depois de um longo período de tempo em um elemento de volume representativo do concreto.
\end{abstract}

Palavras-chave: Reação álcali-sílica, Concreto, Equações de difusão

\section{Introdução}

Desde 1940 muitos estudiosos vêm dedicando suas pesquisas ao estudo de uma patologia do concreto chamada reação álcali-agregado $[7,5,6,11,4]$. A reação álcali-agregado é uma reação que pode vir a danificar o concreto fazendo com que o mesmo tenha sua resistência diminuída. Dentre as reações álcali-agregado, a mais conhecida e também mais comum é a reação álcalisílica. Esta ocorre quando no concreto existe agregado reativo, juntamente com a disponibilidade de álcalis provenientes do cimento Portland e também um alto grau de umidade. A umidade torna os álcalis e hidroxilas disponíveis para quebrar as ligações do tipo siloxano e silanol que estão presentes no agregado reativo. Com a quebra inicia-se a formação de um gel que absorve água e expande.

Enquanto existir espaço na região porosa próxima ao agregado (região de transição) para acomodar o gel, não ocorrerá fissuras. A partir do momento que este espaço torna-se insuficiente, a tensão aumenta e inicia-se a fissuração do concreto. 
Muitos trabalhos tem sido feitos no sentido de criar modelos que tenham como resultado a expansão causada pela reação álcali-sílica $[1,13,2,10,3]$. Os modelos criados associam a expansão devida aos seguintes fatores: umidade, concentração de álcalis, reatividade do agregado e temperatura. Alguns modelos contemplam todos os fatores e outros apenas alguns deles. Entretanto, apesar dos diversos modelos criados não existe um modelo completo capaz de prever a expansão em termos dos fatores citados acima.

Este trabalho trata-se da proposta de um modelo para representar a formação e difusão do gel formado pela reação álcali-silica no concreto. Primeiramente é criado um modelo utilizando a cinética das reações químicas. Este modelo inicial é formado por equações obtidas da reação álcali-sílica, onde é considerada como uma reação de pseudo-primeira ordem, neste caso dependendo da quantidade de sílica disponível. Essas equações permitem obter a quantidade de gel formado ao longo do tempo. A segunda fase é o modelo de difusão para o transporte do gel na região porosa próxima ao agregado. O modelo de difusão leva em conta a concentração de gel formada ao longo do tempo obtido na primeira fase. Como resultado obtém-se um mapa da distribuição do gel em um elemento de volume representativo do concreto. O modelo proposto encontra-se na seção seguinte.

\section{Modelo Proposto}

Este modelo proposto foi criado em duas fases. Na primeira fase é criada uma função para determinar a quantidade de gel formado com a quebra das ligações siloxano. A segunda fase constitui de um modelo difusivo, levando em consideração a quantidade de gel formado na primeira fase.

\subsection{Fase I - Formação do Gel}

A formação do gel é baseada em duas reações principais que são descritas pelas equações:

$$
\begin{gathered}
\equiv \mathrm{Si}-\mathrm{O}-\mathrm{Si} \equiv+\mathrm{R}^{+}+\mathrm{OH}^{-} \rightarrow \equiv \mathrm{Si}-\mathrm{O}-\mathrm{R}+\mathrm{H}-\mathrm{O}-\mathrm{Si} \equiv, \\
\equiv \mathrm{Si}-\mathrm{OH}+\mathrm{R}^{+}+\mathrm{OH}^{-} \rightarrow \equiv \mathrm{Si}-\mathrm{O}-\mathrm{R}+\mathrm{H}_{2} \mathrm{O} .
\end{gathered}
$$

Partindo dessas equações, observa-se que o gel $(\equiv \mathrm{Si}-\mathrm{O}-\mathrm{R})$ é formado nas duas fases da reação. Desta maneira, utilizando os princípios da cinética química, pode-se escrever as seguintes equações:

$$
\begin{aligned}
\frac{d[\mathrm{~A}]}{d t} & =-k_{1}[\mathrm{~A}][\mathrm{B}]_{0}[\mathrm{C}]_{0} \\
\frac{d\left[\mathrm{X}_{\mathrm{sil}}\right]}{d t} & =k_{1}[\mathrm{~A}][\mathrm{B}]_{0}[\mathrm{C}]_{0}-\mathrm{k}_{2}\left[\mathrm{X}_{\mathrm{sil}}\right][\mathrm{B}]_{0}[\mathrm{C}]_{0} \\
\frac{d\left[\mathrm{X}_{\text {gel }}\right]}{d t} & =k_{1}[\mathrm{~A}][\mathrm{B}]_{0}[\mathrm{C}]_{0}+\mathrm{k}_{2}\left[\mathrm{X}_{\mathrm{sil}}\right][\mathrm{B}]_{0}[\mathrm{C}]_{0}
\end{aligned}
$$

onde $[\mathrm{A}]$ é a concentração de sílica ( $\equiv \mathrm{Si}-\mathrm{O}-\mathrm{Si} \equiv)$, $[\mathrm{B}]_{0}$ é a concentração de íons hidroxila $\left(\mathrm{OH}^{-}\right),[\mathrm{C}]_{0}$ é a concentração de álcalis (sódio ou potássio), $\left[\mathrm{X}_{\text {gel }}\right]$ é a concentração de gel formada, $\left[\mathrm{X}_{\text {sil }}\right]$ é a concentração de silanol $(\equiv \mathrm{Si}-\mathrm{OH})$ formado, $k_{1}$ e $k_{2}$ são constantes de velocidade.

Neste modelo deveria ser considerado as taxas de variação para os íons hidroxila e também para os álcalis. Entretanto a existência de ambos é uma condição necessária para que a reação ocorra. Logo, por hipótese para este modelo, considera-se as respectivas concentrações como constantes ao longo do tempo, o que caracteriza essa reação como uma reação de pseudoprimeira ordem, da mesma maneira como é considerado no trabalho de Kurtis et al. [8]. Observe que esta é uma simplificação proposta para o modelo, uma vez que trabalhar com reações de terceira ordem tornaria o modelo excessivamente complexo. 
A equação (3) retrata que a velocidade com que a sílica é consumida é proporcional ao produto das concentrações de íons hidroxila, álcalis e sílica presentes em um instante de tempo $t$.

Na equação (4), pode-se observar que a concentração de silanol ao longo do tempo é proporcional a sua formação na primeira etapa da reação química (1) e pelo consumo na segunda etapa (2).

$\mathrm{Na}$ formação do gel, representado pela equação (5), observa-se que o gel é formado nas duas etapas da reação química.

Como as quantidade $B$ (íons hidroxila) e $C$ (álcalis) foram considerados constantes ao longo do tempo, obtém-se um sistema de equações diferenciais de primeira ordem:

$$
\frac{d}{d t}\left[\begin{array}{c}
{[\mathrm{A}]} \\
{\left[\mathrm{X}_{\mathrm{sil}}\right]} \\
{\left[\mathrm{X}_{\mathrm{gel}}\right]}
\end{array}\right]=\left[\begin{array}{ccc}
-\alpha & 0 & 0 \\
\alpha & -\beta & 0 \\
\alpha & \beta & 0
\end{array}\right]\left[\begin{array}{c}
{[\mathrm{A}]} \\
{\left[\mathrm{X}_{\mathrm{sil}}\right]} \\
{\left[\mathrm{X}_{\mathrm{gel}}\right]}
\end{array}\right]
$$

onde $\alpha=k_{1}[\mathrm{~B}]_{0}[\mathrm{C}]_{0}$ e $\beta=k_{2}[\mathrm{~B}]_{0}[\mathrm{C}]_{0}$ para simplificação. As condições iniciais para o problema são: $[\mathrm{A}](0)=\mathrm{A}_{0}$, ou seja, a quantidade inicial de sílica disponível no início da reação é dada por $A_{0}$. Sendo o agregado reativo este possuí uma quantidade inicial de ligações do tipo silanol, ou seja, a concentração de silanol inicial é $\left[\mathrm{X}_{\text {sil }}\right](0)=\mathrm{X}_{0}$. A concentração inicial de gel é igual a zero, ou seja, $\left[\mathrm{X}_{\text {gel }}\right](0)=0$.

Resolvendo o sistema acima utilizando autovalores e autovetores, encontra-se os autovalores para a matriz associada ao sistema: $\lambda_{1}=-\alpha, \lambda_{2}=-\beta$ e $\lambda_{3}=0$, associado aos autovetores $v_{1}=[(\beta-\alpha) / \alpha, 1,(\alpha-2 \beta) / \alpha]^{\prime}, v_{2}=[0,-1,1]^{\prime}$ e $v_{3}=[0,0,1]^{\prime}$ que fornecem a solução para o sistema:

$$
\begin{aligned}
{[\mathrm{A}](\mathrm{t}) } & =A_{0} e^{-\alpha t} \\
{\left[\mathrm{X}_{\mathrm{sil}}\right](t) } & =\frac{k_{1} A_{0}}{k_{2}-k_{1}}\left(e^{-\alpha t}-e^{-\beta t}\right)+X_{0} e^{-\beta t} \\
{\left[\mathrm{X}_{\text {gel }}\right](t) } & =A_{0}\left[2+\frac{1}{k_{2}-k_{1}}\left[\left(k_{1}-2 k_{2}\right) e^{-\alpha t}+k_{1} e^{-\beta t}\right]\right]+X_{0}\left(1-e^{-\beta t}\right)
\end{aligned}
$$

Observe que neste modelo criado foi considerado apenas uma quebra da ligação siloxano. As outras não foram consideradas, embora ocorram também.

As constantes de proporcionalidade devem ser determinadas a partir de dados obtidos experimentalmente. Neste trabalho as constantes foram obtidas utilizando-se os resultados experimentais fornecidos no trabalho de Larive [9]. Os valores obtidos são: $k_{1}=0,1$ e $k_{2}=0,2$ para uma temperatura média de $38^{\circ} \mathrm{C}$.

No gráfico 1 observa-se que a concentração de gel é uma curva crescente que se aproxima de $2 A_{0}+X_{0}$. Isto se justifica pelo fato de que o consumo de íons é proporcional ao consumo de sílica, ou seja, na proporção de 1:1. Com a concentração inicial de silanol, completa-se a concentração final de gel que é de $2 A_{0}+X_{0}$.

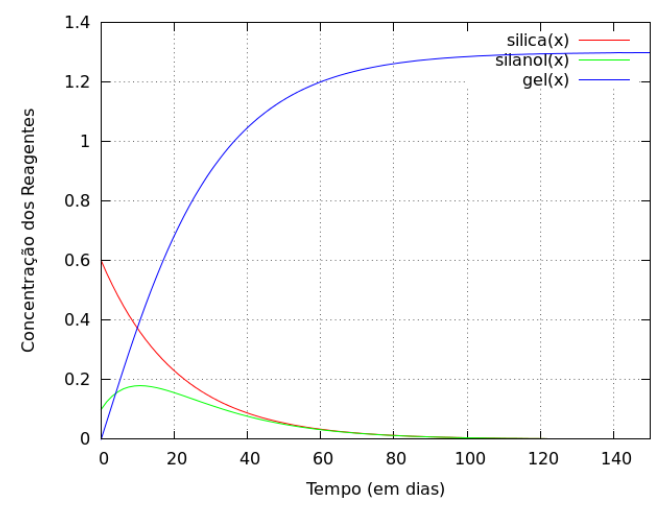

Figura 1: Representação gráfica das funções para formação do gel e consumo da sílica e silanol 


\subsection{Fase II - Difusão do gel}

A modelagem para a difusão do gel na matriz de concreto é feito a um nível mesoscópico, ou seja, o processo difusivo será considerado no agregado graúdo e na região de transição próxima ao agregado. Serão considerados dois modelos distintos, uma vez que o agregado pode se localizar em uma região que esteja com os poros contendo água e outro onde os poros estão sujeitos apenas a um gradiente de umidade.

No primeiro caso, como a quantidade de água é abundante será considerado apenas o processo difusivo do gel em uma matriz porosa contendo líquido no interior dos seus poros.

Considerando um meio onde há uma mistura binária de duas espécies A (gel) e B (matriz porosa contendo água no interior dos poros), no qual a aproximação de um meio estacionário se aplica, ou seja, a transferência de massa ocorre apenas por difusão, onde a advecção é considerada desprezível, obtém-se a equação

$$
\nabla\left(\rho D_{A B} \nabla G\right)+\dot{n}_{A}=\frac{\partial \rho_{A}}{\partial t}
$$

onde $G$ é a fração mássica da espécie $\mathrm{A}$ (gel), $D_{A B}$ é difusividade mássica binária $\mathrm{em} \mathrm{m}^{2} / \mathrm{s}$, $\rho_{A}$ é a massa específica da espécie A (gel) em $\mathrm{kg} / \mathrm{m}^{3}, \rho$ é a massa específica, $\mathrm{kg} / \mathrm{m}^{3}, \dot{n}_{A}$ é a taxa mássica de aumento da espécie A por unidade de volume devido a reações químicas em $\mathrm{kg} /\left(\mathrm{s} \cdot \mathrm{m}^{3}\right)$.

A equação acima pode ser resolvida para fornecer a distribuição de concentrações da espécie A.

Neste modelo, a espécie A será o gel formado pela reação química. Como o meio por onde o gel se difunde é poroso, pode-se alterar a equação de difusão acima para

$$
\nabla\left(\rho D_{A B} \frac{\epsilon_{p}}{\tau} \nabla G\right)+\dot{n}_{A}=\frac{\partial \rho_{A}}{\partial t}
$$

onde

$\epsilon_{p}$ é a porosidade da partícula - adimensional;

$\tau$ é a tortuosidade - adimensional;

A taxa mássica de aumento da espécie $\mathrm{A}, \dot{n}_{A}$ neste caso será dado pela quantidade de gel produzida na primeira etapa do modelo, ou seja:

$$
n_{A}=\left[\mathrm{X}_{\mathrm{gel}}\right](t)=A_{0}\left[2+\frac{1}{k_{2}-k_{1}}\left[\left(k_{1}-2 k_{2}\right) e^{-\alpha t}+k_{1} e^{-\beta t}\right]\right]+X_{0}\left(1-e^{-\beta t}\right)
$$

No segundo caso, a região de transição assim como o agregado estarão sujeitos a umidade. Neste caso será considerado um modelo difusivo para a água na região. A equação é semelhante a equação de difusão do gel, com a diferença que não ocorre formação ou perda de água:

$$
\nabla\left(\rho D_{A B} \frac{\epsilon_{p}}{\tau} \nabla H\right)=\frac{\partial \rho_{A}}{\partial t}
$$

onde $H$ é a concentração de água na matriz porosa.

A reação química ocorre quando tem-se umidade alta em torno do agregado (supondo que o agregado é reativo e há íons alcalinos disponíveis). Como citado anteriormente, a reação ocorre quando os níveis de umidade estão acima de $80 \%$. Sendo assim, a equação anterior dará a concentração de água na região ao longo do tempo. Dependo da quantidade de água disponível ocorrerá a formação e difusão do gel. Este segundo processo difusivo será tratado com a equação (11).

A forma do agregado em ambos os casos serão considerados como elípticos e obtidos a partir de uma curva granumétrica no trabalho de Pianezzer [12].

Para resolver estes modelos numericamente, as equações são discretizadas em seu domínio pelo método das diferenças finitas.

Como resposta final tem-se a concentração de gel na região mesoscópica considerada. Essa concentração poderá ser utilizada para estipular a tensão e deformação associadas ao processo químico. 


\subsection{Resultados preliminares}

A equação (11) foi discretizada pelo Método das Diferenças Finitas explicíto para duas dimensões. Considerando que $\rho_{A}=\rho G$ obteve-se a seguinte discretização:

$$
\frac{G_{i, j}^{n+1}-G_{i, j}^{n}}{\Delta t}=D_{A B} \frac{\epsilon_{p}}{\tau}\left[\frac{G_{i+1, j}^{n}-2 G_{i, j}^{n}+G_{i-1, j}^{n}}{(\Delta x)^{2}}+\frac{G_{i, j+1}^{n}-2 G_{i, j}^{n}+G_{i, j-1}^{n}}{(\Delta y)^{2}}\right]+\frac{n_{a_{i, j}^{n+1}}^{n+} n_{a_{i, j}^{n}}^{n}}{\rho \Delta t}
$$

onde,

$$
\frac{\partial G}{\partial t} \approx \frac{G_{i, j}^{n+1}-G_{i, j}^{n}}{\Delta t}, \quad \frac{\partial^{2} G}{\partial x^{2}} \approx \frac{G_{i+1, j}^{n}-2 G_{i, j}^{n}+G_{i-1, j}^{n}}{(\Delta x)^{2}} \text { e } \frac{\partial^{2} G}{\partial y^{2}} \approx \frac{G_{i+1, j}^{n}-2 G_{i, j}^{n}+G_{i-1, j}^{n}}{(\Delta y)^{2}} .
$$

Fazendo $s_{x}=\frac{D_{A B} \epsilon_{p} \Delta t}{\tau(\Delta x)^{2}}$ e $s_{y}=\frac{D_{A B} \epsilon_{p} \Delta t}{\tau(\Delta y)^{2}}$, tem-se

$$
G_{i, j}^{n+1}=G_{i, j}^{n}+s_{x}\left[G_{i+1, j}^{n}-2 G_{i, j}^{n}+G_{i-1, j}^{n}\right]+s_{y}\left[G_{i, j+1}^{n}-2 G_{i, j}^{n}+G_{i, j-1}^{n}\right]+\frac{n_{a_{i, j}^{n+1}}^{n}-n_{a_{i, j}^{n}}^{n}}{\rho}
$$

Resolvendo a equação (16) numericamente para um tempo de 10 anos, obtém-se os seguintes mapas de distribuição do gel.
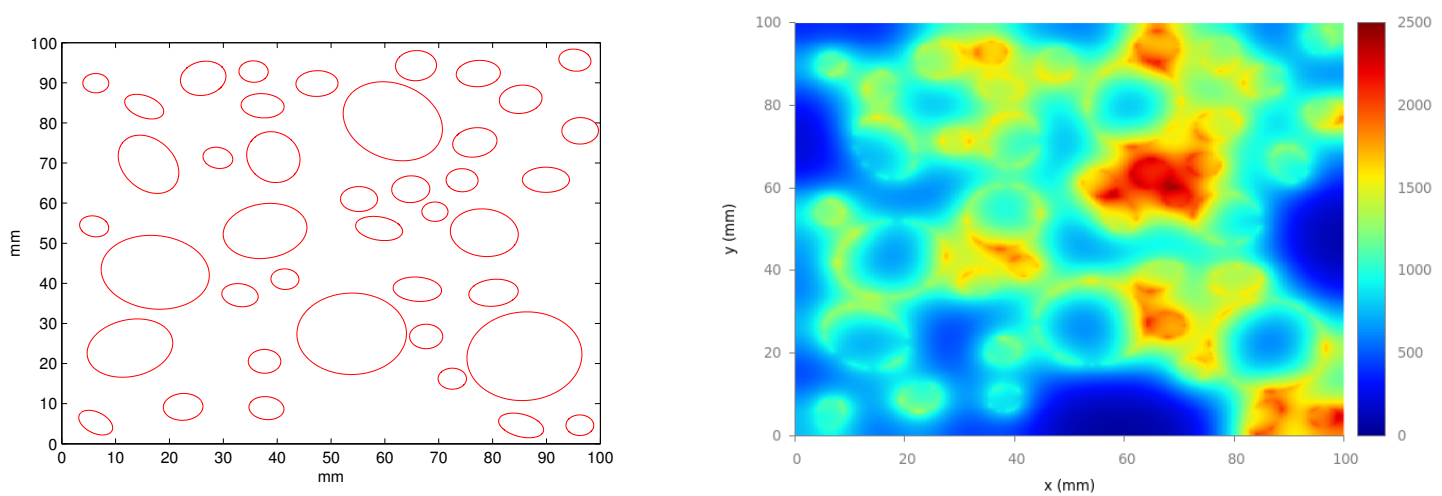

Figura 2: Acima o RVE com agregados elípticos com 30\% de área e abaixo a distribuição da concentração de gel após 1 ano
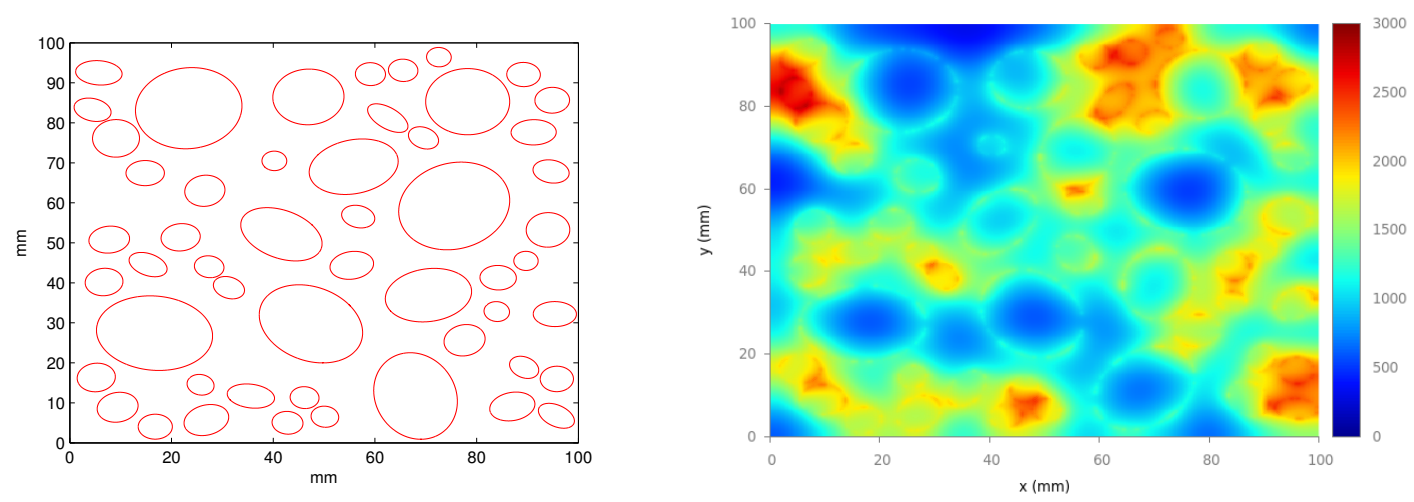

Figura 3: A esquerda o RVE com agregados elipticos com $40 \%$ de área e a direita a distribuição da concentração de gel após 1 ano 

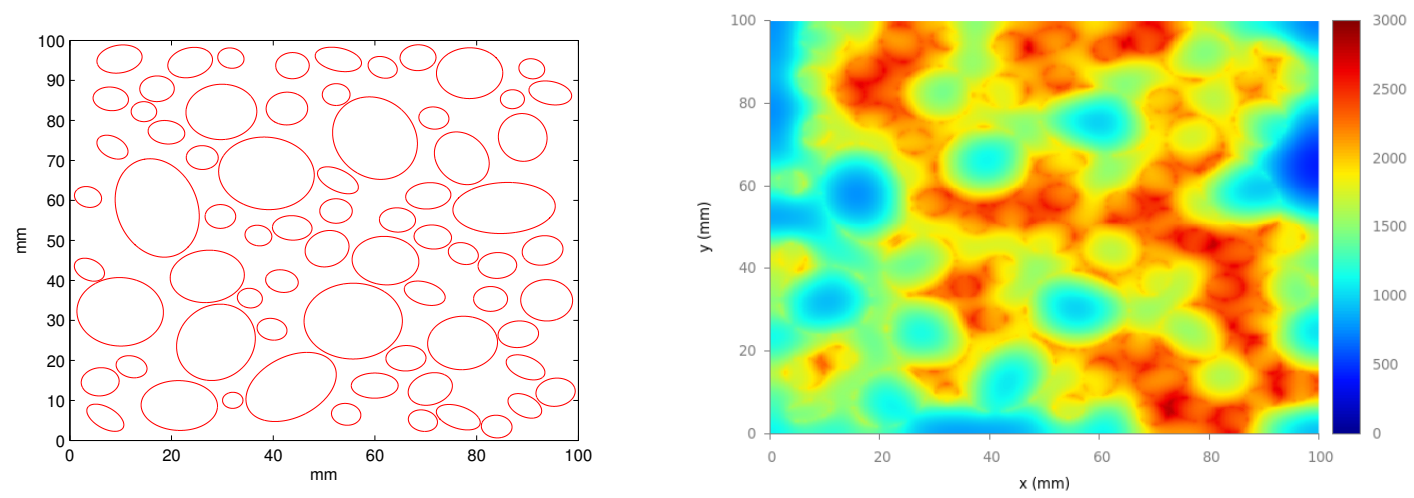

Figura 4: A esquerda o RVE com agregados elipticos com 50\% de área e a direita a distribuição da concentração de gel após 1 ano

Estes resultados foram obtidos para a difusão apenas do gel formado na superfície do agregado, levando em consideração que a curva foi ajustada para a variação de volume obtida por Larive [9] com a temperatura constante de 38 graus Celsius.

As constantes de difusão para o gel foram $D_{A B}=10^{-10} \mathrm{~m}^{2} / \mathrm{s}$ na argamassa e $D_{A B}=10^{-12}$ $\mathrm{m}^{2} / \mathrm{s}$ no agregado, uma vez que não há resultados experimentais para esse tipo de difusão. O coeficiente de difusão do gel no agregado é menor que o coeficiente de difusão na argamassa, uma vez que a porosidade é maior na região de transição agregado/argamassa. A difusão de água foi considerado como constante, pois após um pequeno período de tempo a concentração de água no elemento representativo de volume tornou-se homogênea.

Nos gráficos 2, 3 e 4 pode-se observar que a concentração de gel é maior na região próxima aos agregados maiores, onde existem agregados menores próximos. Uma vez que este trabalho encontra-se em fase de desenvolvimento, não foram considerados resultados experimentais para a validação do modelo. A próxima etapa deste trabalho será a realiazação de experimentos afim de validar ou realizar alterações no modelo existente.

\section{Conclusão}

Este trabalho apresenta um modelo para formação e difusão do gel em um elemento representativo de volume do concreto. Dados experimentais devem ser analisados afim de tornar este modelo representativo para a RAS. Esses resultados experimentais serão considerados na próxima etapa deste trabalho.

\section{Referências}

[1] Bazant, Z. P., Steffens, A., 2000. A mathematical model for kinetics of alkali-silica reaction in concrete. Cemente and Concrete Research 30 (3), 419-428.

[2] Comby-Peirot, I., Bernard, F., Bouchard, P. O., Bay, F., Garcia-Diaz, E., 2009. Development and validation of a $3 \mathrm{~d}$ computational tool to describe concrete behavior at mesoscale. application to the alkali-silica reaction. Computational Material Science 46 (4), 1163-1177.

[3] Comi, C., Fedele, R., Perego, U., 2009. A chemo-thermo-damage model for the analysis of concrete dams affected by alkali-silica reaction. Mechanics of Material 41 (3), 210-230.

[4] Dron, R., Brivot, F., 1992. Thermodynamic and kinetic approach to the alkali-silica reaction. part 1: Concepts. Cement and Concrete Research 22 (5), $941-948$. 
[5] Glasser, L., 1979. Osmotic pressure and the swelling of gels. Cement and Concrete Research $9(4), 515-517$.

[6] Glasser, L. D., Kataoka, N., 1981. Some observations on the rapid chemical test for potentially reactive aggregate. Cement and Concrete Research 11 (2), 191 - 196.

[7] Hobbs, D. W., 1981. The alkali-silica reaction - a model for predicting expansion in mortar. Magazine of Concrete Research 33 (117), 208 - 220.

[8] Kurtis, K. E., Collins, C. L., Monteiro, P. J. M., 2002. The surface chemistry of the alkalisilica reaction: a critical evaluation and x-ray microscopy. Concret Science and Engineering $4,2-11$.

[9] Larive, C., 1997. Apports combinés de l'expérimentation et de la modélisation la comprehénsion de l'alcali-réaction et de ses effets méchaniques. Ph.D. thesis, École Nationale des Ponts et Chausses, Paris, France.

[10] Pan, J. W., Feng, Y. T., Wang, J. T., Sun, Q. C., Zhang, C. H., Owen, D. R. J., 2012. Modelling of alkali-siica reaction in concrete: a review. Front. Struct. Civ. Eng 6 (1), 1-18.

[11] Pesavento, F., Gawin, D., Wyrzykowski, M., Schrefler, B. A., Simoni, L., 2012. Modeling alkali-silica reaction in non-isothermal, partially saturated cement based materials. Computer Methods in Applied Mechanics and Engineering 225-228 (0), 95 - 115.

[12] Pianezzer, G. A., 2013. Geração do elemento representativo do concreto com agregados graúdos no formato elíptico. Dissertação de mestrado, UFPR - Universidade Federal do Paraná, Curitiba, PR.

[13] Suwito, A., Jin, W., Xi, Y., Meyer, C., 2002. A mathematical model for the pessimum effect of asr in concrete. Concrete Science and Engineering 4, 23-34. 